\title{
PHYSIOLOGICAL MEASUREMENT ON STUDENTS' ENGAGEMENT IN A DISTRIBUTED LEARNING ENVIRONMENT
}

\author{
Chen Wang ${ }^{1}$ and Pablo Cesar ${ }^{1}$ \\ ${ }^{I}$ Centrum Wiskunde \& Informatica, Amsterdam, the Netherlands \\ $\{c w, p . s . c e s a r\} @ c w i . n l$
}

Keywords: E-learning; surveys; GSR sensors; students' engagement

\begin{abstract}
Measuring students' engagement in a distributed learning environment is a challenge. In particular, a teacher gives a lecture at one location, while at the same time the remote students watch the lecture through a display screen. In such situation, it is difficult for the teacher to know the reaction at the remote location. In this paper, we conducted a field study to measure students' engagement by using galvanic skin response (GSR) sensors, where students simultaneously watched the lecture at the two locations. Our results showed the students' GSR response was aligned with the surveys, which means that during a distributed learning environment, GSR sensors can be used as an indicator on students' engagement. Furthermore, our user studies resulted in non-engaging student learning experiences that would be difficult obtained at a lab condition. Based on the findings, we found that the patterns of GSR readings were rather different when compared to the previous relevant studies, where users were engaged. In addition, we noticed that the density of GSR response at the remote location was higher when compared to the one at the lecture room. We believe that our studies are beneficial on physiological computing, as we first presented the patterns of GSR sensors on non-engaging user experiences. Moreover, as an alternative method, GSR sensors can be easily implemented in a distributed learning environment to provide feedback to teachers.
\end{abstract}

\section{INTRODUCTION}

E-learning technology has effectively changed the lecture paradigm, and has provided more flexibility to let people choose their preferable time to follow recorded lectures (Foertsch, Moses, Strikwerda and Litzkow, 2002). Furthermore, the pace of technology for use in computing education is staggering, as we have seen, during the last five years, the following tools/ websites have completely transformed the way of teaching: Piazza, Google Docs, YouTube, Doodle and whenisgood.net, Skype and Google Hangout (Garcia and Segars, 2012).

In previous studies on E-learning, researchers have conducted experiments for assessing learning facilities: a blackboard and a hangout platform (Erkollar, Alptekin and Oberer, 2013), comparisons between Hangout and an existing E-learning platform (Strudler and Grove, 2013). Some of studies have used surveys to measure the usability of an E-learning platform (Zhang, Rui, Crawford and He, 2008; Faulkner and McClelland, 2002), and to develop solutions to enhance the learners' experience (Wang, Chen, Liu and Liu 2009). Few studies used physiological sensors to evaluate the students' biofeedback to interactive and noninteractive material (Wirtky, Laumer, Eckhardt, and Weitzel, 2013), i.e., discriminant analysis was applied to extract sensor data as a feature generator. As Kaiser et al. stated (Kaiser and Oertel, 2006), an emotion recognition sensor system can enhance Elearning system by adding affective abilities.

However, there are some issues that are not addressed in the previous studies. First, users may respond differently under a lab condition when compared to a field study (Fairclough, 2009). In particular, Fairclough claimed that physiological response manipulated in a lab could not be reproduced in naturalistic settings. Even though a lab study might be sufficient for testing how 

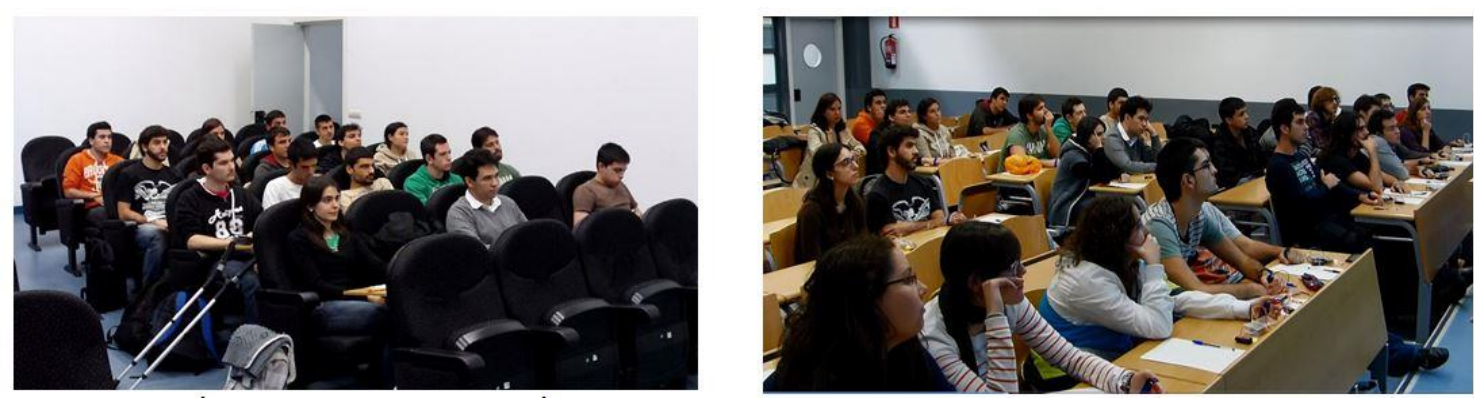

Figure 1: Students at two locations: (left) the students joined the lecture, and (right) the students watched the lecture through Google+ Hangout.

students learn alone, that is not the case for actual learning, as students might react different in the class. Second, measuring students' engagement towards a lecture is different than evaluating an interaction method or a usability test. Evaluating an interaction method or conducting a usability test can be done in several rounds, but in the case of measuring students' engagement to a real lecture, repeating experiments may cause different styles of teacher presentation. The different presentation styles may generate the different response of students. Last, we believe that a ground truth, e.g. a survey, is required to understand the patterns of physiological sensors, especially when such patterns are linked to user engagement.

In this paper, we conducted a field study to measure students' engagement in an E-learning environment, where GSR sensors captured the students' biofeedback. During the experiment, one location was a lecture classroom with the teacher, and the other location was a remote classroom where students followed the lecture in a project screen (Figure $1 \&$ Figure 3 ). After that, we compared the sensor results with the results from the surveys. We are in particular interested in the following research question:

R1: Can GSR sensors be used to measure students' engagement in an E-learning environment?

By answering the research question, we may deploy GSR sensors on E-learning platform. Without the need of surveys, we can use GSR sensors to monitor students' engagement and provide feedback to the teacher.

In our experiment, we interpret students' GSR response as engagement. We restricted our research topic to the scope of E-learning.

This paper is structured as follows. First, we discuss the related work. Then, we describe the experimental design, detailing the data collection, data analysis, and the participants. Next, we report our results regarding the questionnaires and the physiological sensors. Last, there are a discussion and conclusion section.

\section{RELATED WORK}

\subsection{Types of studies on E-learning}

We can divide the studies on E-learning on four main types: E-learning platform development, Elearning platform usability test, E-learning material evaluation, and E-learning interaction method development. Traditionally, E-learning platforms were developed based on video -conferencing systems (Zhang, Rui, Crawford and He, 2008; Faulkner and McClelland, 2002). Recently, some new technologies have been incorporated in order to large amount of students, e.g., cloud computing (Aljenaa, Anzi and Alshayeji, 2011). Open corpus resources made the learning materials accessible anyway, anytime, and from any device (Christina and Cabriel, 2009). Furthermore, some methods related to affective computing have been applied in E-learning environment to enhance learners' engagement. For example, empathic virtual human or social software can be included on an E-learning platform to increase learners' performance (Wirtky, Laumer, Eckhardt and Weitzel, 2013). Last, some studies have focused on the usability test of an Elearning platform (Alsumait and Osaimi, 2009).

Surveys and physiological sensors are the main methods in terms of evaluating the usability of system, the suitability of learning materials, or the performance of learners. For example, Faulkner et al. used surveys to investigate how a video conferencing system can deliver an educational program to women consumers in rural and remote area (Faulkner and McClelland, 2012). Clark et al. used surveys to study whether the social platform Google+ had a better performance on developing teaching material when compared to a text-based Elearning platform (Neal and Grove, 2013). Furthermore, both Handri et al. and Brawner et al applied physiological sensors to evaluate the impacts 

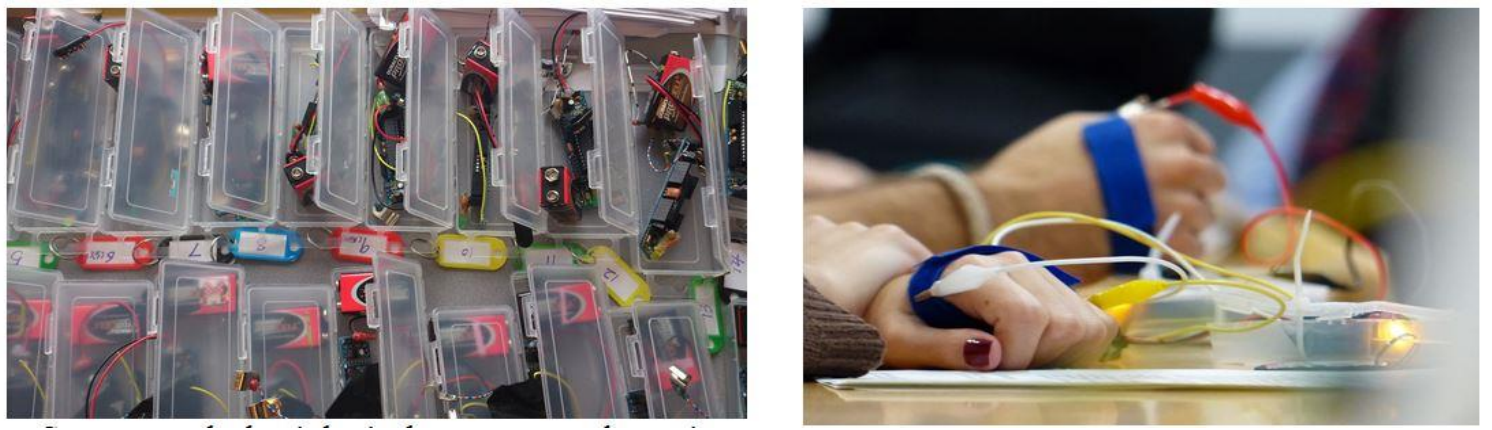

Figure 2: The GSR sensors. The left picture shows the whole set of GSR sensors, and the right picture shows how the GSR sensors were worn during the experiment.

of course materials and user response towards a computer-based training system (Handri, Yajima, Nomura, Ogawa, Kurosawa and Fukumura, 2010; Brawner and Goldberg, 2012). In addition, facial recognition was also applied to detect learners' emotional states, so that a virtual tutor could provide effective feedback based on these emotional cues (Dmello and Graesser, 2013).

\subsection{GSR Sensors}

GSR sensors, also known as galvanic skin response (GSR), electrodermal response (EDR), psychogalvanic reflex (PGR), skin conductance response (SCR), or skin conductance level (SCL). GSR sensors measure users' electrical conductance of the skin, where users' sweat glands are varied and controlled by the sympathetic nervous system. Therefore, GSR sensors are normally considered as an indicator of psychological or physiological arousal. When users are highly aroused, users' skin conductance is increased in turn. Furthermore, in affective computing and HCI, GSR sensors have been proved as a valid approach for measuring audience engagement, and researchers have shown interesting results between GSR and engagement (Mandryk, 2003; Picard, 1997).

As for our knowledge, few studies used GSR sensors to evaluate the learners' performance on Elearning environment (Brawner and Goldberg, 2012). However, GSR sensors, combined with other sensors, have been extensively applied on some other scenarios, e.g., video gaming and theatre performance. For instance, based on physiological signals, Ruan et al. proposed a discriminant model to predict the fatigue state of players, so that the design of body-controlled games can be adapted and improved (Ruan, Chen, Sun and Chen, 2009). In addition, GSR sensors have also been studied for performing arts, Latuliper et al. and Chen et al. used both surveys and GSR sensors to investigate audience engagement at a lab and a field study respectively ( Latulipe, Carroll and Lottridge, 2011; Wang, Geelhoed, Stenton and Cesar, 2014).

\section{EXPERIMENTAL DESIGN}

\subsection{Participants}

There were 17 students at each location: the lecture classroom with four females and thirteen males (Mean age $=21.05, \mathrm{SD}=2.16$ ), and the remote classroom six females and eleven males (Mean age $=22.29, \mathrm{SD}=2.02$ ). The experiment was conducted at a scheduled class - the last class on Structured Query Language (SQL) database before an exam, and both the teacher and the students did not have any experience on sensor experiments before (Figure 1). During the lecture, there was no interaction between the teacher and the students. Before the experiment started, they signed a consent form for the video recordings. After the experiment, all the students received a small gift as a bonus.

\subsection{Questionnaires}

Before the experiment, we conducted a prequestionnaire in order to examine the students' physical condition, emotional state, and daily learning habit. After the experiment, the students took a small exam in order to check the learning outcomes. Afterwards, they filled out a short questionnaire about their learning experience during the lecture. All of the questions were in the form of "Graphic Rating Scales" in which participants were asked to make a mark on a line between two extremes, e.g.

How much did you enjoy during the lecture? 


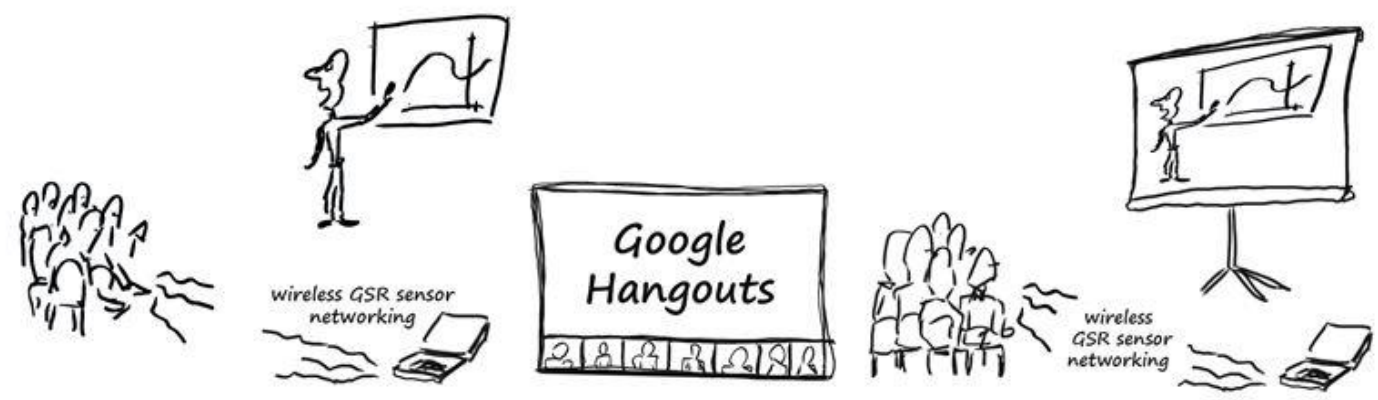

Figure 3: System architecture: the left is the lecture classroom; the middle is the Google+ Hangout platform, streaming the lecture into the remote classroom; the right is the remote classroom.

Not at all
The line measured $100 \mathrm{~mm}$ and responses were
measured to $1 \mathrm{~mm}$ accurate.

\subsection{Steaming Technology}

We chose Google+ Hangout as the streaming platform for the remote students. The reason is that such platform has been considered as a capable and low-cost solution that can be used in education and training. For instance, Erkollar et al. studied the impact of using Google+ and a blackboard respectively (Erkollar, Alptekin and Oberer, 2013). Similarly, Clark et al. conducted an experiment to investigate whether the affordances of Google+ would more effectively help develop teaching and enhance social presence when compared with the university's current text-based WebCT discussion platform (Neal and Grove, 2013).

\subsection{Methodologies}

Questionnaires ratings and GSR readings were analysed using Analysis of Variance (ANOVA), correlations and Multi-Dimensional Scaling (MDS). At each location, the repeat measurements were used on the pre- and post- questionnaires, to examine the impact of the lecture. In terms of the different experience between the two locations, the betweensubjects design was used for both surveys and GSR sensor data.

Multidimensional scaling (MDS) is a means of visualizing the level of similarity of individual cases of a dataset, in particular to display the information contained in a distance matrix (Yound and Hamer, 1987; Schiffman, Reynolds and Young, 1981). Furthermore, MDS technique aims to place each object in $\mathrm{N}$-dimensional space such that the between-object distances are preserved as well as possible. In our analysis, we used a two dimensional space to display the similarities between the averaged audience GSR responses.

MDS has been widely applied in psychological research (Trevor and Cox, 2000; Borg and Groenen, 2002), but it is another new research technique to physiological computing. Unlike other statistical techniques that test hypotheses that have been proposed a priori, MDS is an exploratory data method that explores data for which no specific hypotheses have been formed. Therefore, we do not require to check the assumptions on the data sets, but we need to report the overall fit statistics (Kruskal's stress and R Square) in the MDS, as they are the indication about how the algorithm fits the input data. In our case, we applied the MDS to visualize the lecture impact on the two locations, so that we could compare the density of GSR response on the two locations.

In the results of Pearson product-moment correlation coefficient, we used one star "*" representing 95\% confidence level and two stars “**” indicating 99\% confidence level.

All the data analysis was done using SPSS. C language and Python were used to develop the hardware and sensor data collection. Before performing the ANOVA, we checked the assumptions (normality and homogeneity of variance) in order to assuring the validation of the results. In our case, our data satisfied these assumptions.4.1 Survey Results (the ground truth)

\section{RESULTS}

\subsection{Survey Results (the ground truth)}


The repeated measurement results showed that the students at the lecture classroom had a significant decrease on cheerful, energy and attention, but sadness and being tired did not change after the lecture (Table 1). While for the remote students, there were no significant differences found after the lecture (Table 2). Furthermore, the students gave the rather low ratings on most of items of the surveys: averaging 4.9 at the lecture classroom and 4.7 at the remote classroom.

By examining the post-questions between the two location students, we found that the students at the lecture classroom were more cheerful but less comfortable when compared to the remote students (Table 3).

\begin{tabular}{|c|c|c|c|}
\hline Item & $\mathbf{p}$ & Mean_pre & Mean_post \\
\hline Cheerful & 0.008 & 6.07 & 4.4 \\
\hline Sad & 0.81 & 2.25 & 2.39 \\
\hline Energy & 0.04 & 5.38 & 4.03 \\
\hline Tired & 0.27 & 3.86 & 3.01 \\
\hline Attention & 0.001 & 6.38 & 3.88 \\
\hline
\end{tabular}

Table 1: The differences between pre- and postquestionnaires at the lecture classroom, and yellow indicates a significant difference found at $\mathrm{p}$ value.

\begin{tabular}{|c|c|c|c|}
\hline Item & p & Mean_pre & Mean_post \\
\hline Cheerful & 0.31 & 5.2 & 5.6 \\
\hline Sad & 0.24 & 2.4 & 3.03 \\
\hline Energy & 0.06 & 6.1 & 5.1 \\
\hline Tired & 0.28 & 3.01 & 3.9 \\
\hline Attention & 0.06 & 6.12 & 4.9 \\
\hline
\end{tabular}

Table 2: The differences between pre- and postquestionnaires at the remote classroom, where no significant values were found at $\mathrm{p}$ value.

\begin{tabular}{|c|c|c|c|}
\hline Items & $\begin{array}{c}\text { Mean(Remote/ } \\
\text { Lecture) }\end{array}$ & p & (R-L)\% \\
\hline Cheerful & $3.59 / 5.82$ & $*$ & -0.38 \\
\hline Comfortable & $6.56 / 2.36$ & $* *$ & 1.78 \\
\hline
\end{tabular}

Table 3: The significant differences found with the surveys between the two locations.

Q1: How much cheerful were you during the lecture?

Q2: How much comfortable were you during the lecture?

The results in Table 3 are very interesting. It seems that the remote students felt more comfortable during the lecture, but they were less cheerful. We believe this is because the remote students could easily direct their attention to other things than the lecture, e.g., checking other classmates' states or mobile phones, as we saw in the video recordings. In contrast, the students at the lecture classroom had to pay attention to the teacher, and thus they felt less comfortable. In terms of the difference on the cheerful state between the two locations, we think that this may be related to the teacher's presence, as the remote students watched the lecture through a screen projector.

\subsection{Exam Results}

The test scores showed the students at both locations had a good performance (Table 4). The reason may be the lecture was an extra lecture, and did not introduce much new knowledge. Furthermore, there was a significant difference on the scores found between the two locations. The students at the lecture classroom achieved a higher score (around $16 \%$ higher) than the remote students. In addition, the pre-questionnaires showed there was no significant difference on the previous knowledge between the two locations.

\begin{tabular}{|c|c|c|c|}
\hline Items & Mean(Remote/Lecture) & p & $\begin{array}{c}\text { (R- } \\
\text { L)\% }\end{array}$ \\
\hline Scores & $7.76 / 9.29$ & $*$ & -0.16 \\
\hline
\end{tabular}

Table 4: The significant differences were found on exam scores between the students at both locations.

\subsection{GSR Sensors Results (R1)}

\subsubsection{Arousal}

We found that the arousal levels at the two locations were both negative (Fig. 4), and these results were aligned with the survey data: both location students were not so much engaged during the lecture. Moreover, we found that the remote students' arousal was lower than the one at the lecture classroom (but the statistical value is at p: 0.06 , which is not significant).

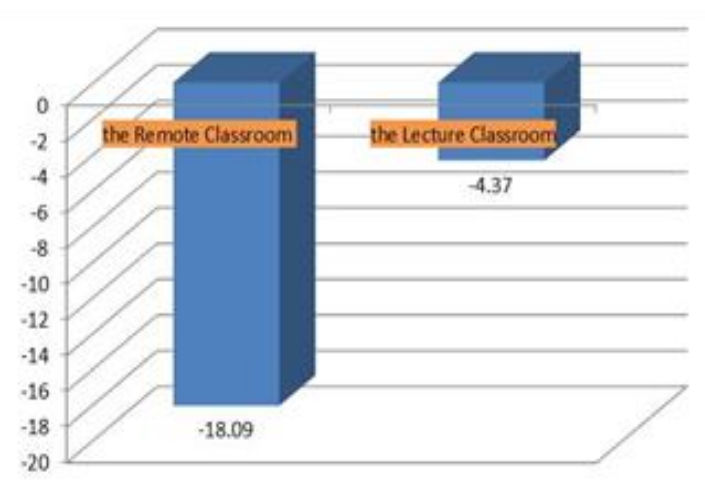

Figure 4: The arousal values at both locations are negative. 


\subsubsection{Correlations}

We found the correlations results on our data sets were rather different to other relevant study that used a brain sensor. Dmochowski et al. found that "when two people watch a movie, their brains respond similarly - but only if the video is engaging. These results were obtained by using a brain wave sensor, while user watched videos available in social media (Dmochowski, Bezdek, Abelson, Johnson, Schumacher and Parra, 2014). In our experiment, we had a non-engaging learning experience, and it is important to note that such situation (non-engaging experience) has not been reported before. Unlike the previous studies, the audience was engaged with a video (Dmochowski, Bezdek, Abelson, Johnson, Schumacher and Parra, 2014) or a comedy play (Wang, Geelhoed, Stenton and Cesar, 2014). However, during a non-engaging experience, we found that there were no such significant correlations existed between the two locations (surveys and GSR response), and there was no significant cross correlation between surveys and GSR readings. In addition, the study conducted by Wang et al., (Wang, Geelhoed, Stenton and Cesar, 2014), 10 of 15 audience members formed a big engaging cluster. Yet, we noticed that in our study, there were several clusters established at each location based on the students' GSR response, and this made it difficult to interpret the each cluster by simply checking surveys.

\subsubsection{The density of GSR response on the two locations}

We displayed the MDS results on the twodimensional map (Figure 5), where each point indicates the averaged minute by minute students' GSR response at the two locations. We found that the density of GSR responses at the two locations was rather different: the GSR response (the green points) on the remote students was more closed to each other when compared to the one (the red points) at the lecture classroom. According to Wang et al. study (Wang, Geelhoed, Stenton and Cesar, 2014), they found the more similar GSR response, the more close distance between the adjacent timelined (based on every minute) points. Therefore, we think this result may imply, during a non-engaging experience, the remote location GSR response was more synchronized (the green points are more closed to each other) when compared to the one (the red points) at the lecture classroom. This might be related to the different user states at the two locations, e.g., comfortable or cheerful, although the students at the two locations were both non-engaged during the lecture.

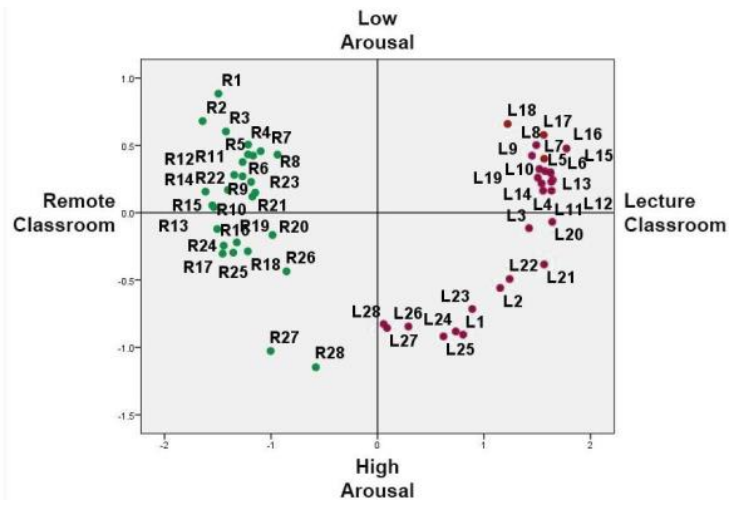

Figure 5: MDS minute by minute unfolding of the lecture impact at the both locations: the density of GSR responses at the remote location was higher when compared to the one at the lecture room $(\mathrm{R}$ : the lecture duration at the remote classroom; L: the lecture duration at the lecture classroom). (Stress: 0.1, RSQ: 0.98)

As Figure 5 displayed, the remote students spent the first 3 minutes to be synchronized on their GSR response - a massive cluster appeared afterwards, but their GSR response started to have a big jump distance at the minute 26 until the end of the lecture. In contrast, the GSR response of the students at the lecture classroom had the similar manner, after the first 3 minutes, there was a big cluster formed on their GSR response from the minute 4 to the minute 20. Unlike the GSR response at the remote location, the GSR response at the lecture classroom had a big jump at the minute 21 , afterwards, there was another cluster established until the end of the lecture.

\section{DISCUSSION}

In this study, we obtained the realistic GSR data on students' engagement in a real lecture, except that the students were required to wear a sensor. The results report a non-engaging user experience, and such results have not been reported before.

We think that a field study might more easily capture a non-engaging user experience, as users are placed in a realistic environment. On the contrary, if users are placed under a lab condition, users normally treat the stimulus as a task, to which they pay attention, as they have to fulfil the assignment we give to them. Furthermore, in a lab condition, even though users label a bored state, their actual biofeedback may be rather different to a real bored state (Fairclough, 2009, Wang and Cesar, 2014). 
Our results on the density of GSR responses at the two locations do not conflict with the previous correlation results, as we mentioned in the part of methodologies, the MDS algorithm is a technique to explore the data. The explored findings will motivate us to make a further investigation on user experiences that are linked to the patterns of GSR sensors.

In addition, we suggest not adding extra task to users during a physiological experiment, i.e., user annotations, as such constant labelling work would distract user experience and alter sensor readings. Therefore, we suggest obtaining a ground truth by some other methods, e.g., surveys or video recordings.

Last, the measurement in our study was simultaneously done at the two locations. However, for the applications E-learning, e.g., watching an educational video, this might not be required. It will be an interesting future work to conduct experiments with these two different experimental settings. In particular, we are interested in investigating how the GSR patterns look like on non-engaging home learners, whether they have the similar/different sensor patterns compared to non-engaging learners who watch a lecture at a classroom. However, the resulted learner' engagement cannot be predicted before the experiment, as we normally obtain such information after the experiment.

\section{CONCLUSION}

In this paper, we used GSR sensors to measure students' engagement in a field study - a distributed learning environment. The experimental results showed that the GSR sensors' measurement was aligned with the surveys, so that we can use GSR sensors as an alternative method to measure students' engagement. Furthermore, we compared the resulted non-engaging user experiences to the previous similar studies, and we found that GSR readings demonstrated the different patterns that have not been reported before. In addition, the MDS result revealed that the GSR response at the remote location was more synchronized when compared to the one at the lecture classroom.

We believe that our study is beneficial for Elearning, as we have shown that GSR sensors can be used as an alternative tool to provide feedback in a distributed learning environment. Moreover, we presented a non-engaging user case, where the patterns of GSR readings were first reported. Last, the methodologies incorporated in this study are also helpful on other sensor studies, e.g., a pulse sensor.

\section{REFERENCESF}

Daniel D. Garcia and Luke Segars. 2012. Technology that educators of computing hail (TECH): come, share your favorites!. In Proceedings of the 43rd ACM technical symposium on Computer Science Education (SIGCSE '12). ACM, New York, NY, USA, 682-682. DOI=10.1145/2157136.2157438

Erkollar, Alptekin, and B. J. Oberer. "Putting Google+ to the Test: Assessing Outcomes for Student Collaboration, Engagement and Success in Higher Education." Procedia-Social and Behavioral Sciences 83 (2013): 185-189.

Strudler, Neal, and Karen Grove. "I see you: Using the affordances of Google+ to increase social and teaching presence in an online undergraduate teacher education course ISTE 2013." Cynthia Clark doctoral student, San Antonio, TX. Retrieved from http://www. isteconference. org/uploads/ISTE2013/HANDOUTS/KEY_80520342/I STE2013ISeeYou_RP.pdf. Accessed on June 17 (2013): 2013.

Chen Wang, Erik N. Geelhoed, Phil P. Stenton, and Pablo Cesar. 2014. Sensing a live audience. In Proceedings of the 32nd annual ACM conference on Human factors in computing systems (CHI '14). ACM, New York, NY, USA, 1909-1912. DOI $=10.1145 / 2556288.2557154$

Young, Forrest W., and Robert M. Hamer (ed.)(1987), Multidimensional Scaling: History,Theory, and Applications, Hillsdale, NJ: Erlbaum.

Schiffman, Susan S., M. Lance Reynolds, and Forrest W. Young (1981), Introduction to Multidimensional Scaling: Theory, Methods, and Applications, NY: Academic Press.

Trevor F. Cox and M.A.A. Cox. Multidimensional Scaling, Second Edition. ISBN 1-58488-094-5.

Ingwer Borg and Patrick J.F. Groenen. Modern Multidimensional Scaling: Theory and Applications. 2005 Springer Science+Buiness Media, Inc. ISBN-10: 0-387-25150-2.

Foertsch, J., Moses, G., Strikwerda, J. and Litzkow, M. (2002), Reversing the Lecture/Homework Paradigm Using eTEACH ${ }^{\circledR}$ Web-based Streaming Video Software. Journal of Engineering Education, 91: 267 274. doi: 10.1002/j.2168-9830.2002.tb00703.x

Mavlankar, A; Agrawal, P.; Pang, D.; Halawa, S.; NgaiMan Cheung; Girod, B., "An interactive region-ofinterest video streaming system for online lecture viewing," Packet Video Workshop (PV), 2010 18th International , vol., no., pp.64,71, 13-14 Dec. 2010 doi: 10.1109/PV.2010.5706821

Cha Zhang, Yong Rui, Jim Crawford, and Li-Wei He. 2008. An automated end-to-end lecture capture and broadcasting system. ACM Trans. Multimedia 
Comput. Commun. Appl. 4, 1, Article 6 (February 2008), 23 pages. DOI=10.1145/1324287.1324293

Kathryn Faulkner and Linda McClelland. (2002). Using videoconferencing to deliver a healthy education program to women healthy consumers in rural and remote queensland: an early attempt and future plans. Aust. J. Rural Health 10, 65-72.

Dmochowski, Jacek P, Bezdek, Matthew A, Abelson, Brian P, Johnson, John S, Schumacher, Eric H, Parra, Lucas C. 2014. Audience preferences are predicted by temporal reliability of neural processing. Nature Publishing Group, a division of Macmillan Publishers Limited. http://dx.doi.org/10.1038/ncomms5567. $\underline{10.1038 / \text { ncomms5567 }}$

Chin-Yeh Wang, Gwo-Dong Chen, Chen-Chung Liu, and Baw-Jhiune Liu. 2009. Design an empathic virtual human to encourage and persuade learners in elearning systems. InProceedings of the first ACM international workshop on Multimedia technologies for distance learning (MTDL '09). ACM, New York, NY, USA, 27-32. DOI=10.1145/1631111.1631117

Cristina Hava Muntean and Gabriel-Miro Muntean. 2009. Open corpus architecture for personalised ubiquitous e-learning. Personal Ubiquitous Comput. 13, 3 (March 2009), 197-205. DOI=10.1007/s00779-007-0189-5

Thomas Wirtky, Sven Laumer, Andreas Eckhardt, and Tim Weitzel. 2013. Using social software for enhancing IS talents' e-learning motivation. In Proceedings of the 2013 annual conference on Computers and people research (SIGMIS-CPR '13). ACM, New York, NY, USA, 63-72. DOI $=10.1145 / 2487294.2487307$

E. Aljenaa, F. S. Al-Anzi, and M. Alshayeji. 2011. Towards an efficient e-learning system based on cloud computing. In Proceedings of the Second Kuwait Conference on e-Services and e-Systems(KCESS '11). ACM, New York, NY, USA, , Article 13, 7 pages. DOI $=10.1145 / 2107556.2107569$

Vladimir Kolovski and John Galletly. 2003. Towards Elearning via the semantic web. InProceedings of the 4th international conference conference on Computer systems and technologies: e-Learning (CompSysTech '03), B. Rachev and A. Smrikarov (Eds.). ACM, New York, NY, USA, 591-596. DOI=10.1145/973620.973719

Asmaa Alsumait and Asma Al-Osaimi. 2009. Usability heuristics evaluation for child e-learning applications. In Proceedings of the 11th International Conference on Information Integration and Web-based Applications \& Services (iiWAS '09). ACM, New York, NY, USA, 425-430. DOI $=10.1145 / 1806338.1806417$

Santoso Handri, Kuniaki Yajima, Shusaku Nomura, Nobuyuki Ogawa, Yoshimasa Kurosawa, and Yoshimi Fukumura. 2010. Evaluation of Student's Physiological Response Towards E-Learning Courses Material by Using GSR Sensor. In Proceedings of the
2010 IEEE/ACIS 9th International Conference on Computer and Information Science (ICIS '10). IEEE Computer Society, Washington, DC, USA, 805-810. DOI=10.1109/ICIS.2010.92

Keith W. Brawner and Benjamin S. Goldberg. 2012. RealTime monitoring of ECG and GSR signals during computer-based training. In Proceedings of the 11th international conference on Intelligent Tutoring Systems (ITS'12), Stefano A. Cerri, William J. Clancey, Giorgos Papadourakis, and Kitty Panourgia (Eds.). Springer-Verlag, Berlin, Heidelberg, 72-77. DOI $=10.1007 / 978-3-642-30950-2 \_10$

Sidney D'mello and Art Graesser. 2013. AutoTutor and affective autotutor: Learning by talking with cognitively and emotionally intelligent computers that talk back. ACM Trans. Interact. Intell. Syst. 2, 4, Article 23 (January 2013), 39 pages. DOI $=10.1145 / 2395123.2395128$

R. Mandryk. Objectively evaluating entertainment technology. In CHI'04, pages 1057-1058. ACM Press, 2003.

R.W. Picard. Affective computing. MIT Press, Cambridge, MA, USA, 1997.

Shengsheng Ruan, Ling Chen, Jie Sun, and Gencai Chen. 2009. Study on the change of physiological signals during playing body-controlled games. In Proceedings of the International Conference on Advances in Computer Enterntainment Technology (ACE '09). ACM, New York, NY, USA, 349-352. DOI=10.1145/1690388.1690456

Celine Latulipe, Erin A. Carroll, and Danielle Lottridge. 2011. Love, hate, arousal and engagement: exploring audience responses to performing arts. In Proceedings of the SIGCHI Conference on Human Factors in Computing Systems (CHI '11). ACM, New York, NY, USA, 1845-1854. DOI=10.1145/1978942.1979210

Stephen H. Fairclough. Fundamentals of physiological computing. Interact. Comput. (2009) 21 (1-2): 133 145.

Robin Kaiser and Karina Oertel. 2006. Emotions in HCI: an affective e-learning system. InProceedings of the HCSNet workshop on Use of vision in humancomputer interaction - Volume 56(VisHCI '06), Roland Goecke, Antonio Robles-Kelly, and Terry Caelli (Eds.), Vol. 56. Australian Computer Society, Inc., Darlinghurst, Australia, Australia, 105-106.

Chen Wang and Pablo Cesar. 2014, Do we react in the same manner? Comparing GSR patterns across scenarios. NordiCHI 2014. 